\title{
Identification of the Time-Dependent Point Source in a System of two Coupled Two Dimension Diffusion-Advection-Reaction Equations: Application to Groundwater Pollution Source Identification
}

\author{
Alpha Omega Soko*a, Okelo Jeconiah Abonyo ${ }^{\mathrm{b}}$, and Verdiana Grace Masanja ${ }^{\mathrm{c}}$ \\ ${ }^{a}$ Department of Mathematics, Pan African University Institute for Basic Sciences \\ Technology and Innovation (PAUSTI), Nairobi, Kenya. \\ ${ }^{\mathrm{b}}$ Department of Pure and Applied Mathematics, Jomo Kenyatta University of \\ Agriculture and Technology (JKUAT), Nairobi, Kenya. \\ ${ }^{\mathrm{c}}$ Nelson Mandela African Institution of Science and Technology (NM-AIST), Arusha, \\ Tanzania.
}

\begin{abstract}
This paper addresses the inverse source problem in a system of two-dimension advection-dispersion reaction equation with an emphasis on groundwater pollution source identification. We develop an inverse source problem method for identifying the unknown groundwater point sources utilizing only the boundary and interior measurements. We develop an identifiability criterion of the point sources from recording the oxygen deficit concentration relative to the biochemical oxygen demand concentration. We have also established an identification method that uses the records of oxygen deficit concentration and biochemical oxygen demand concentration to identify the source position as a solution to nonlinear dispersion current equations. We recover the source intensity function using the multi-dimension inverse Laplace transform of the de-convolution function without any need of an iterative process. The inverse Laplace transforms are approximated by shifted Legendre Polynomials. The results show that the proposed inverse problem method is accurate.
\end{abstract}

Keywords: Groundwater pollution source, Inverse source Problem, Advection Dispersion Reaction Equation, Laplace Transform, Shifted Legendre Polynomials.

\footnotetext{
${ }^{*}$ Corresponding author: Alpha Omega Soko.

E-mail:alpha@aims.ac.tz
} 


\section{INTRODUCTION}

To measure groundwater quality, it is necessary to measure the composition of organic matter mixed in the groundwater. There are many ways to measure the composition of organic matter in water. Two of the methods used are Chemical Oxygen Demand (COD) and Biochemical Oxygen Demand (BOD). BOD has been a useful indicator to measure groundwater quality. BOD measures the amount of dissolved oxygen required or consumed for the microbiological decomposition (oxidation) of organic material in water[10, 1].

When measuring BOD, it requires five days due to laboratory treatment for the results to be available [7]. The five days of waiting makes pollution source identification impossible before this period. However, for some applications such as alerting a drinking aquifer station about accidental groundwater pollution, it is essential to reduce this waiting time in order to be effective since after the five days the conditions may have changed and responding to the measured BOD may not be appropriate. This marks the main objective of the present work, where the aim is to identify pollution sources using the records of the Dissolved Oxygen (DO) concentration which have the advantage to be available immediately. The mathematical modelling involves the BOD concentration coupled with the Oxygen Deficit Concentration (ODC). The ODC is obtained as the difference between the saturation DO concentration and the actual DO concentration in the groundwater.

The present study is motivated by the results established in the papers $[1,6]$. In [1], an inverse source problem with only one 2D transport equation was analysed, which correspond to the identification of pollution sources from recording the BOD concentration data. We extend the work reported in [6] to the two-dimension system. Unlike the one-dimension model of [6], we are interested in the inverse source problem that consists of the identification of a spatial-temporal varying point source involved in a system of two coupled 2D linear transport equations.

The originality of the present study consists in addressing the linear inverse source problem of identifying unknown spatial-temporal point sources occurring in a 2D coupled Advection Dispersion Reaction Equation (ADRE). Although the 2D mathematical model is subject to a lot of interest, the identification of spatial-temporal point sources in the 2D coupled evolution transport equations remains an open problem.

The paper is organized as follows: Section 2 is devoted to stating the problem, assumptions and proving a few technical results for later use. In Section 3, we construct Green's functions to use on the intensity identification. Section 4 is reserved to establish an identification method that uses the ODC-BOD records to identify the elements defining the point source. In Section 4.1 we provide methodology for the identification of intensity function using the de-convolution function and the inverse Laplace transform. The inverse Laplace transforms are approximated by the Shifted Legendre polynomials (SLPs) in Section 4.1.1. Section 4.2 is devoted to source identification by locating roots of dispersion current non-linear system of equations using Newton's Method. Some 
numerical experiments on the groundwater pollution BOD-ODC coupled model are presented in Section 5. We end the paper by providing the conclusion in Section 6.

\section{GOVERNING EQUATION AND PROBLEM FORMULATION}

The model concerns the measurement of pollutant concentration by the use of $B O D_{5}$ values and ODC values denoted herein by $C$ and $\zeta$ respectively. Let $\Omega \in \mathbb{R}^{2}$ be any connected open set with boundary $\partial \Omega$. The boundary $\partial \Omega$ is the union of four disjoint boundaries, that is, $\partial \Omega=\Gamma_{1} \cup \Gamma_{2} \cup \Gamma_{3} \cup \Gamma_{4}$ where $\Gamma_{1}$ and $\Gamma_{4}$ represents the inflow and outflow boundaries respectively and $\Gamma_{2} \cup \Gamma_{3}$ represents the lateral lower and upper boundaries. Denote by $\Gamma_{L}$ the union of lateral boundaries, that is, $\Gamma_{L}=\Gamma_{2} \cup \Gamma_{3}$. The BOD-ODC model is governed by the system of two coupled 2D linear Equations (2.1) and (2.2) [10]:

$$
\begin{aligned}
& \frac{\partial \zeta}{\partial t}-\nabla \cdot(D \nabla \zeta-\mathbf{V} \cdot \zeta)+R_{1} \zeta=R_{1} C \\
& \frac{\partial C}{\partial t}-\nabla \cdot(D \nabla C-\mathbf{V} \cdot C)+R_{2} C=F(x, y, t)
\end{aligned}
$$

where $C, \zeta$ are BOD and ODC concentrations measured in $m g / L$ respectively, $\mathbf{V}$ is the flow velocity measured in $m / s, R_{1}$ and $R_{2}$ are the reaction coefficients measured in $s^{-1}$ , $F$ represents the set of all occurring pollution sources measured in $m g L^{-1} s^{-1}$ and $D$ denotes the hydrodynamic dispersion tensor measured in $\mathrm{m}^{2} / \mathrm{s}$.

Since the Equations (2.1) and (2.2) are the equations for the groundwater flow, they can be further simplified by applying the following assumptions; firstly, the groundwater is incompressible. Mathematically, this means that the density, $\rho$, of the groundwater is constant and therefore the continuity equation takes Equation (2.3):

$$
\frac{\partial \rho}{\partial t}+\nabla \cdot(\rho \mathbf{V})=0 \quad \Longleftrightarrow \nabla \cdot \mathbf{V}=0
$$

secondly, $\mathbf{V}$ satisfies the no slip boundary conditions which means that along lateral boundaries, the groundwater has zero velocity relative to the boundary, that is, Equation (2.4):

$$
\mathbf{V}=0 \quad \text { on } \quad \Gamma_{L} \times(0, T)
$$

Thirdly, the groundwater flow is irrotational meaning the flow with zero vorticity or net rate of change of angular velocity in all directions is zero for the flow. Mathematically, irrotational flow occurs when the cross gradient of the velocity is zero, that is, Equation (2.5).

$$
\nabla \times \mathbf{V}=0 \quad \text { in } \quad \Omega \times(0, T)
$$

Simplifying the Equations (2.1) and (2.2) using the Equation (2.3) gives Equations (2.6) 
and (2.7);

$$
\begin{aligned}
& \frac{\partial \zeta}{\partial t}+\mathbf{V} \cdot \nabla \zeta-\nabla \cdot(D \nabla \zeta)+R_{1} \zeta=R_{1} C \\
& \frac{\partial C}{\partial t}+\mathbf{V} \cdot \nabla C-\nabla \cdot(D \nabla C)+R_{2} C=F(x, y, t)
\end{aligned}
$$

For the reaction term, we use the BOD-DO relationships introduced by the United States Environmental Protection Agency (USEPA) under the QUAL I model [11]. The reaction term of the Equation (2.7) is the same as the reaction term from [4] that is, the Equation (2.9). For ODC in Equation (2.6) it is given by Equation (2.8):

$$
\begin{aligned}
R_{1}([D O]) & =K_{a}\left([D O]_{s}-[D O]\right)-\left(K_{d}+K_{s}\right)[B O D] \\
R_{2}([B O D]) & =\left(K_{d}+K_{s}\right)[B O D]
\end{aligned}
$$

where $[D O]_{s}$ represents the concentration of saturated dissolved oxygen and $K_{a}, K_{d}, K_{s}$ are the re-aeration, de-oxygenation and sedimentation rates respectively.

Hydrodynamic dispersion refers to the stretching of a solute band in the flow direction during its transport by an advecting fluid [12]. It occurs as a consequences of two processes; molecular diffusion which results from the random molecular motion and Mechanical dispersion which is caused by non uniform velocities. With these two processes the hydrodynamic dispersion tensor is given by Equation (2.10):

$$
D=\left(\begin{array}{cc}
D_{x x} & D_{x y} \\
D_{y x} & D_{y y}
\end{array}\right)
$$

where the spatially varying entries $D_{x x}, D_{y y}, D_{x y}, D_{y x}$ are such that Equations (2.11) to $(2.13)$ holds $[2,9]$ :

$$
\begin{aligned}
& D_{x x}=\frac{a_{L} v_{1}^{2}+a_{T} v_{2}^{2}}{\|\mathbf{V}\|^{2}} \\
& D_{y y}=\frac{a_{T} v_{1}^{2}+a_{L} v_{2}^{2}}{\|\mathbf{V}\|^{2}} \\
& D_{x y}=\frac{\left(a_{L}-a_{T}\right) v_{1} v_{2}}{\|\mathbf{V}\|^{2}}=D_{y x}
\end{aligned}
$$

Where $a_{T}$ and $a_{L}$ are the transverse and longitudinal dispersion coefficients respectively and

$$
\|\mathbf{V}\|=\sqrt{v_{1}^{2}+v_{2}^{2}}
$$

As for the source function, $F(x, y, t)$, we consider time-dependent point sources of the 
Equation (2.14);

$$
F(x, y, t)=w(t) \delta\left(x-S_{x}, y-S_{y}\right)
$$

where $w(t)$ is the source intensity function, $\delta$ is the Dirac delta function, $\left(S_{x}, S_{y}\right)$ is the source location. For the initial condition, without loss of generality, the assumption that no pollution occurs at the initial monitoring time is reasonable since at this time the pollutant has yet to mix with the water. Thus a null initial BOD concentration. Since, the pollutants are introduced on the boundaries not in the interior an assumption that no pollution concentration on the rectangular domain of $\partial \Omega$ is reasonable. Finally, a null gradient concentration at the downstream boundary which represent no variation in BOD changes. Therefore, in view of the Equations (2.6) and (2.7), the BOD-ODC concentrations satisfies the Equations (2.15) to (2.19):

$$
\begin{aligned}
\frac{\partial \zeta}{\partial t}+\mathbf{V} \cdot \nabla \zeta-\nabla \cdot(D \nabla \zeta)+R_{1} \zeta & =R_{1} C \\
\frac{\partial C}{\partial t}+\mathbf{V} \cdot \nabla C-\nabla \cdot(D \nabla C)+R_{2} C & =w(t) \delta\left(x-S_{x}, y-S_{y}\right) \\
C(x, y, 0)=\zeta(x, y, 0) & =0 \quad \text { in } \quad \Omega \\
C(x, y, t)=\zeta(x, y, t) & =0 \quad \text { on } \quad \Gamma_{1} \times(0, T) \\
\nabla C(x, y, t)=\nabla \zeta(x, y, t) & =0 \quad \text { on } \quad\left(\Gamma_{L} \cup \Gamma_{4}\right) \times(0, T)
\end{aligned}
$$

The problem in the Equations (2.15) to (2.19) admits a unique solution denoted here by the couple $(C, \zeta)$ that belongs to the functional space given by Equation (2.20)[8];

$$
C \in L^{2}\left(0, T ; L^{2}(\Omega)\right) \cap \mathscr{C}\left(0, T ; H^{-1}(\Omega)\right) \quad \text { and } \quad \zeta \in \mathscr{C}\left(0, T ; L^{2}(\Omega)\right)
$$

As the source position, $\left(S_{x}, S_{y}\right)$, is assumed to be in the interior of the domain $\Omega$, the state $\zeta$ is smooth on the boundary $\partial \Omega$, which allows to define the boundary observation operator in the Equation (2.21):

$$
M[F]:=\{\zeta(a, t), \zeta(b, t) \text { for } 0<t<T\}
$$

where $a$ and $b$ are observation points satisfying $0<a<b<l$. This is called the direct problem. The inverse problem the paper is dealing with is: assuming available the records

$$
\left\{d_{a}(t), d_{b}(t) \quad \text { for } \quad 0<t<T\right\}
$$

of the concentration $C(x, y, t)$ at the two observation points $a$ and $b$, find the source function, $F$, such Equation (2.23)

$$
M[F]=\left\{d_{a}(t), d_{b}(t) \quad \text { for } \quad 0<t<T\right\}
$$

Theorem 2.1. Let $F_{i}=w_{i}(t) \delta\left(x-S_{x}^{i}, y-S_{y}^{i}\right)$, for $i=1,2$ where $w_{i}(t)$ is a positive 
function of $L^{2}(0, T)$. Assume that the source positions $\left(S_{x}^{i}, S_{y}^{i}\right)$ are distinct. If $M\left(F_{1}\right)=M\left(F_{2}\right)$, then $w_{1}=w_{2}$ and $S_{x}^{1}=S_{x}^{2}, S_{y}^{1}=S_{y}^{2}$.

Proof. Assume that the source positions $\left(S_{x}^{i}, S_{y}^{i}\right)$ for $i=1,2$ are distinct. Let

$$
F_{i}=w_{i}(t) \delta\left(x-S_{x}^{i}, y-S_{y}^{i}\right), \quad \text { for } \quad i=1,2
$$

be the two sources which are giving rise to the same boundary observations. Let $C_{i}, \zeta_{i}$ be the corresponding solutions of the Equations (2.15) to (2.19). Assume that $M\left(F_{1}\right)=M\left(F_{2}\right)$, we have to show that $F_{1}=F_{2}$. Denote the differences $C_{2}-C_{1}$ and $\zeta_{2}-\zeta_{1}$ by $\varphi$ and $\vartheta$ respectively. Clearly $(\varphi, \vartheta)$ are solutions to the following system of equations

$$
\begin{aligned}
\frac{\partial \vartheta}{\partial t}+\mathbf{V} \cdot \nabla \vartheta-\nabla \cdot(D \nabla \vartheta)+R_{1} \vartheta & =R_{1} \varphi \\
\frac{\partial \varphi}{\partial t}+\mathbf{V} \cdot \nabla \varphi-\nabla \cdot(D \nabla \varphi)+R_{2} \varphi & =w_{2}(t) \boldsymbol{\delta}\left(\mathbf{x}-\mathbf{S}_{2}\right)-w_{1}(t) \boldsymbol{\delta}\left(\mathbf{x}-\mathbf{S}_{1}\right) \\
\varphi(x, y, 0)=\vartheta(x, y, 0) & =0 \quad \text { in } \quad \Omega \\
\varphi(x, y, t)=\vartheta(x, y, t) & =0 \quad \text { on } \quad \Gamma_{1} \times(0, T) \\
\nabla \varphi(x, y, t)=\nabla \vartheta(x, y, t) & =0 \quad \text { on } \quad\left(\Gamma_{L} \cup \Gamma_{4}\right) \times(0, T)
\end{aligned}
$$

As the coefficients of the Equations (2.24) to (2.28) are all real analytic, then according to Cauchy-Kovalevskaya theorem [13, p.15], the system of the Equations (2.24) to (2.28) has a unique solution near zero. The remaining task is to show that the solution vanishes on the neighbourhood of $\partial \Omega$. Because of the delta function, we know that $\varphi$ is identically zero in $\Omega \backslash \bigcup\left\{S_{1}, S_{2}\right\}$, thus by Mizohata unique continuation theorem [14], we have $\varphi=0$ in $\Omega \backslash \bigcup\left\{S_{1}, S_{2}\right\}$. Since $F_{i} \in H^{-1-\varepsilon}$ for $\varepsilon>0$ then $\varphi \in L^{2}(\Omega)$. Thus $\varphi=0$ on $\Omega$ and eventually, $\vartheta=0$. That implies $F_{1}=F_{2}$ which is equivalent to having $w_{1}=w_{2}$ and $S_{1}=S_{2}$.

For later use, we introduce SLPs due to [15], which are necessary in the inversion of Laplace Transforms. The SLPs are orthogonal polynomials defined by the recurrence on Equation (2.29)

$$
\begin{cases}Z_{0}(t) & =1 \\ Z_{1}(t) & =2 \exp (-t / T)-1 \\ Z_{2}(t) & =6 \exp (-2 t / T)-6 \exp (-t / T)+1 \\ \vdots & \vdots \\ Z_{n+1}(t)= & \frac{2 n+1}{n+1}[2 \exp (-t / T)-1] Z_{n}(t)-\frac{n}{n+1} Z_{n-1}(t) \quad n \geq 1\end{cases}
$$


where $T$ is an arbitrary constant. The shifted Legendre polynomials have the orthogonal property given by Equation (2.30)

$$
\int_{0}^{\infty} Z_{n}(t) Z_{m}(t) \exp (-t / T) \mathrm{dt}= \begin{cases}0, & n \neq m \\ \frac{T}{2 n+1}, & n=m\end{cases}
$$

Any real function $u(t) \in L^{2}(\Omega)$, can be expressed as a finite number of terms of the SLPs as the Equation (2.31)

$$
u(t)=\sum_{i=0}^{N-1} \alpha_{i} \exp (a t) Z_{i}(t)
$$

where $\exp (a t)$ is the weighting function and the expansion coefficient $\alpha_{i}$ is computed by Equation (2.32)

$$
\alpha_{i}=\frac{2 i+1}{T} \int_{0}^{\infty} \exp [-(a+1 / T) t] u(t) Z_{i}(t)
$$

The Equation (2.32) is obtained by minimizing the mean square error in the Equation (2.33)

$$
\varepsilon=\int_{0}^{\infty}\left[u(t)-\sum_{i=0}^{N-1} \alpha_{i} \exp (a t) Z_{i}(t)\right]^{2} \mathrm{dt}
$$

The Laplace Transform of a function $u(t)$ is given by the Equation (2.34)

$$
U(s)=\int_{0}^{\infty} \exp (-s t) u(t) \mathrm{dt}
$$

Making substitution of Equations (2.29), (2.30) and (2.34) into the Equation (2.32), for $i=2,3, \ldots, N-1$ and $n=1,2, \ldots i-1$, we obtain the shifted Legendre expansion coefficient as the Equation (2.35)

$$
\alpha_{i}=\frac{2 i+1}{T} \sum_{n=0}^{i} \sigma_{i, n} U\left(a+\frac{n+1}{T}\right)
$$

where the coefficients $\sigma_{i n}$ are computed recursively using the Equation (2.36)

$$
\begin{aligned}
\sigma_{0,0} & =1, \sigma_{1,0}=-1, \sigma_{1,1}=2, \sigma_{i, 0}=-\sigma_{i-1,0}, \sigma_{i, i}=\left[2(2 i-1) \sigma_{i-1, i-1}\right] \cdot i^{-1} \\
\sigma_{i, n} & =\left[(2 i-1)\left(2 \sigma_{i-1, n-1}-\sigma_{i-1, n}\right)-(i-1) \sigma_{i-2, n}\right] \cdot i^{-1}
\end{aligned}
$$




\section{GREENS FUNCTION METHOD TO SOLVE DIRECT PROBLEM}

Theorem 3.1. Let $\mathbf{G}=\left(G_{1}, G_{2}\right)$ be the Green's Function of the Equations (2.15) to (2.19). Then $\mathbf{G}$ satisfies:

$$
\begin{aligned}
-\frac{\partial G_{1}}{\partial t}-\mathbf{V} \cdot \nabla G_{1}-\nabla \cdot D \nabla G_{1}+R_{1} G_{1} & =\delta(x-\xi) \delta(y-\eta) \delta(t-\tau) \\
-\frac{\partial G_{2}}{\partial t}-\mathbf{V} \cdot \nabla G_{2}-\nabla \cdot D \nabla G_{2}+R_{2} G_{2} & =\delta(x-\xi) \delta(y-\eta) \delta(t-\tau) \\
G_{1}(x, y, 0 ; \xi, \tau, \eta) & =G_{2}(x, y, 0 ; \xi, \tau, \eta)=0 \quad \text { in } \quad \Omega \times(0, T) \\
G_{1}(x, y, t ; \xi, \tau, \eta) & =G_{2}(x, y, t ; \xi, \tau, \eta)=0 \quad \text { on } \quad\left(\Gamma_{1} \cup \Gamma_{4}\right) \times(0, T) \\
\nabla G_{1}(x, y, t ; \xi, \tau, \eta) & =\nabla G_{2}(x, y, t ; \xi, \tau, \eta)=0 \quad \text { on } \quad \Gamma_{L} \times(0, T)
\end{aligned}
$$

Proof. Let $\mathbf{G}=\left(G_{1}, G_{2}\right)$ be the Green's function and let $\mathscr{L}_{1}$ and $\mathscr{L}_{2}$, be the linear parabolic partial differential operators defined by the Equations (3.6) and (3.7):

$$
\begin{aligned}
& \mathscr{L}_{1}[\zeta]=\frac{\partial C}{\partial t}+\mathbf{V} \cdot \nabla C-\nabla \cdot(D \nabla C)+R_{1} C \\
& \mathscr{L}_{2}[C]=\frac{\partial \zeta}{\partial t}+\mathbf{V} \cdot \nabla \zeta-\nabla \cdot(D \nabla \zeta)+R_{2} \zeta
\end{aligned}
$$

The non-homogeneous Equations (2.15) to (2.19) is equivalent to the Equations (3.8) to (3.12):

$$
\begin{aligned}
\mathscr{L}_{1}[\zeta] & =R_{1} C \\
\mathscr{L}_{2}[C] & =w(t) \boldsymbol{\delta}(\mathbf{x}-\mathbf{S}) \\
C(x, y, 0)=\zeta(x, y, 0) & =0 \quad \text { in } \quad \Omega \\
C(x, y, t)=\zeta(x, y, t) & =0 \quad \text { on } \quad \Gamma_{1} \times(0, T) \\
\nabla C(x, y, t)=\nabla \zeta(x, y, t) & =0 \quad \text { on } \quad\left(\Gamma_{L} \cup \Gamma_{4}\right) \times(0, T)
\end{aligned}
$$

Let $u_{1}=C$ and $u_{2}=\zeta$. For $i=1,2$, the adjoint operator, $\mathscr{L}^{*}{ }_{i}$ for the Equations (3.8) and (3.9) satisfies the Green's Identity given by the Equation (3.13):

$$
\int_{0}^{T} \int_{\Omega} G_{i} \mathscr{L}_{i}\left[u_{i}\right] \mathrm{d} \sigma_{\xi} \mathrm{d} \eta=\int_{0}^{T} \int_{\partial \Omega} \sum_{\alpha=1}^{2} \frac{\partial}{\partial \xi_{\alpha}} M_{\alpha}\left(u_{i}, G_{i}\right) \hat{\mathbf{n}} \mathrm{d} \sigma_{\xi} \mathrm{d} \eta+\int_{0}^{T} \int_{\Omega} u_{i} \mathscr{L}^{*}{ }_{i}\left[G_{i}\right] \mathrm{d} \sigma_{\xi} \mathrm{d} \eta
$$

Where $\mathrm{d} \sigma_{\xi}$ is the area element, $\mathrm{d} \eta$ is the time element and the function $M\left(u_{i}, G_{i}\right)$ is a boundary term which involves the values of $u_{i}, G_{i}$, and some of their partial derivatives on the boundary. The boundary terms in the Equation (3.13) and $\mathscr{L}_{i}^{*}$ are found by 
carrying out the integration by parts of the left-hand side with the given operator $\mathscr{L}_{i}$. At the same time, $\mathscr{L}_{i}[C]=w(t) \boldsymbol{\delta}(\mathbf{x}-\mathbf{S})$ and $\mathscr{L}_{i}[\zeta]=R C$, this gives Adjoint operator in the Equations (3.14) and (3.15);

$$
\begin{aligned}
& \mathscr{L}_{1}^{*}\left[G_{1}\right]=-\frac{\partial G_{1}}{\partial t}-\mathbf{V} \cdot \nabla G_{1}-\nabla \cdot D \nabla G_{1}+R_{1} G_{1} \\
& \mathscr{L}_{2}^{*}\left[G_{2}\right]=-\frac{\partial G_{2}}{\partial t}-\mathbf{V} \cdot \nabla G_{2}-\nabla \cdot D \nabla G_{2}+R_{2} G_{2}
\end{aligned}
$$

The boundary terms are given by the Equation (3.16):

$$
\begin{aligned}
\int_{0}^{T} \int_{\partial \Omega} \sum_{\alpha=1}^{2} \frac{\partial}{\partial \xi_{\alpha}} M_{\alpha}\left(u_{i}, G_{i}\right) \cdot \hat{\mathbf{n}} \mathrm{d} \sigma_{\xi} \mathrm{d} \eta & =\int_{0}^{T} \int_{\partial \Omega} u_{i} G_{i} \cdot \hat{\mathbf{n}} \mathrm{d} \sigma_{\xi} \mathrm{d} \eta+\int_{0}^{T} \int_{\partial \Omega} G_{i}\left(\mathbf{V} u_{i}-D \nabla u_{i}\right) \cdot \hat{\mathbf{n}} \mathrm{d} \sigma_{\xi} \mathrm{d} \eta \\
& +\int_{0}^{T} \int_{\partial \Omega} u_{i}\left(D \nabla G_{i}\right) \cdot \hat{\mathbf{n}} \mathrm{d} \sigma_{\xi} \mathrm{d} \eta
\end{aligned}
$$

Depending on the choice of $G$, the Equation (3.13) provides the solution to the original problem. Specifically, if, $G$, satisfies the Equations (3.17) and (3.18):

$$
\begin{aligned}
\mathscr{L}_{1}^{*}\left[G_{1}\right] & =\delta(\mathbf{x}-\xi) \delta(t-\tau) \\
\mathscr{L}_{2}^{*}\left[G_{2}\right] & =\delta(\mathbf{x}-\xi) \delta(t-\tau)
\end{aligned}
$$

Then for $i=1,2$, the last term in Equation (3.13) is $C$ and $\zeta$ respectively. The boundary conditions of Equations (3.17) and (3.18) are obtained from Equation (3.16). Applying boundary conditions in the Equations (3.10) to (3.12) on Equation (3.16) gives the result.

\section{METHOD FOR SOLVING THE INVERSE PROBLEM}

\subsection{Identification of the Unknown Source Intensity Function}

For $i=1,2$, with the Green's function satisfying Theorem 3.1, the left-hand side of Equation (3.13) is just the integral of the greens function multiplied by the functions: $w(t) \delta(\mathbf{x}-\mathbf{S})$ and $R C$ respectively. Thus the solution of the Equations (2.15) to (2.19) is given by the Equations (4.1) and (4.2):

$$
\begin{aligned}
& C(x, y, t)=\int_{0}^{T} \int_{\Omega} G_{1}(x, y, t ; \xi, \eta, \tau) w(\tau) \delta\left(\xi-S_{x}, \eta-S_{y}\right) \mathrm{d} \xi \mathrm{d} \eta \mathrm{d} \tau \\
& \zeta(x, y, t)=\int_{0}^{T} \int_{\Omega} G_{2}(x, y, t ; \xi, \eta, \tau) R_{1} C(\xi, \eta, \tau) \mathrm{d} \xi \mathrm{d} \eta \mathrm{d} \tau
\end{aligned}
$$


Before proceeding with the solution method, we first provide the definition of multi-dimension Laplace transform of a function due to [3];

Definition 4.1. Let $f(x, y, t)$ be a complex valued function of three real variables $x, y$, and, $t$ defined on the plane $(0 \leq x<\infty),(0 \leq y<\infty),(0 \leq t<\infty)$. Then the 3 -dimensional Laplace transform of the function $f(x, y, t)$ is defined in the Equation (4.3)

$$
F(r, p, q)=\int_{0}^{\infty} \int_{0}^{\infty} \int_{0}^{\infty} e^{-r x-p y-q t} f(x, y, t) \mathrm{dxdydt}
$$

Similarly, the inverse of the 3-dimensional Laplace transform $F(r, p, q)$ is given by the Bromwich integral

$$
f(x, y, t)=\frac{1}{(2 \pi i)^{3}} \int_{\alpha_{1}-i \infty}^{\alpha_{1}+i \infty} \int_{\alpha_{2}-i \infty}^{\alpha_{2}+i \infty} \int_{\alpha_{3}-i \infty}^{\alpha_{3}+i \infty} F(r, p, q) e^{r x+p y+q t} \mathrm{drdqdp}
$$

where the integration is done along the vertical plane $\mathfrak{R}(r, p, q)=\left(\alpha_{1}, \alpha_{2}, \alpha_{3}\right)$ in the complex plane such that $\left(\alpha_{1}, \alpha_{2}, \alpha_{3}\right)$ is greater than the real part of all singularities of $F(r, p, q)$ and $F(r, p, q)$ is bounded on the plane.

Immediate use of the Definition 4.1, Theorem 4.2 follows which is about BOD generation from utilizing the oxygen deficit, ODC.

Theorem 4.2. Let, $r, p, q$, be the complex numbers, $G_{2}(x, y, t ; r, p, q)$ and $\zeta(r, p, q)$ be the three-dimension Laplace transform of $G_{2}(x, y, t ; x, y, t)$ and $\zeta(x, y, t)$ respectively, that is, Equation (4.5).

$$
\begin{cases}G_{2}(x, y, t ; r, p, q) & =\int_{0}^{\infty} \int_{0}^{\infty} \int_{0}^{\infty} e^{-q \tau-p \eta-r \xi} G_{1}(x, y, t ; \xi, \eta, \tau) d \xi d \eta d \tau \\ \zeta(r, p, q) & =\int_{0}^{\infty} \int_{0}^{\infty} \int_{0}^{\infty} e^{-q \tau-p \eta-r \xi} \zeta(\xi, \eta, \tau) d \xi d \eta d \tau\end{cases}
$$

The BOD concentration, $C(x, y, t)$, is given by the Equation (4.6)

$$
C(x, y, t)=\frac{1}{(2 \pi i)^{3}} \int_{\alpha_{1}-i \infty}^{\alpha_{1}+i \infty} \int_{\alpha_{2}-i \infty}^{\alpha_{2}+i \infty} \int_{\alpha_{3}-i \infty}^{\alpha_{3}+i \infty}\left(\frac{\zeta(r, p, q) \cdot p \cdot q \cdot r}{R_{1} G_{2}(x, y, t ; r, p, q)}\right) e^{r x+p y+q t} \mathrm{drdqdp}
$$

Proof. Applying the three-dimension Laplace transform of Equation (4.2) both sides and using the Laplace transform convolution theorem gives Equation (4.7)

$$
\zeta(r, p, q)=\frac{R_{1}}{p q r} G_{2}(x, y, t ; r, p, q) C(r, p, q)
$$

Making $C(r, p, q)$ subject of the formulae and talking three-dimension inverse Laplace transform in the Equation (4.7) gives the result.

Since $C(x, y, t)$ has been determined by Theorem 4.2 , the source intensity function 
$w(t)$ is found by solving Equation (4.1) using the $C(x, y, t)$. The result is given by Theorem 4.3.

Theorem 4.3. Let, $q$, be a complex number, $G_{1}\left(x, y, t ; S_{x}, S_{y}, q\right)$ and $C(x, y, q)$ be the Laplace Transform of $G_{1}(x, y, t ; \xi, \eta, \tau)$ and $C(x, y, t)$ respectively, that is, Equation (4.8)

$$
\left\{\begin{array}{l}
G_{1}\left(x, y, t ; S_{x}, S_{y}, q\right)=\int_{0}^{\infty} e^{-q \tau} G_{1}(x, y, t ; \xi, \eta, \tau) d \tau \\
C(x, y, q)=\int_{0}^{\infty} e^{-q \tau} C(x, y, \tau) d \tau
\end{array}\right.
$$

provided that the Laplace Transforms exist, then the intensity function $w(t)$ is given by the Equation (4.9)

$$
w(t)=\frac{1}{2 \pi i} \int_{\alpha-i \infty}^{\alpha+i \infty}\left(\frac{C(x, y, q)}{G_{1}\left(x, y, t ; S_{x}, S_{y}, q\right)}\right) e^{q t} \mathrm{dq}
$$

Proof. Simplifying the Equation (4.1) by using the dirac delta shifting property gives the Equation (4.10)

$$
C(x, y, t)=\int_{0}^{T} w(\tau) G_{1}\left(x, y, t ; S_{x}, S_{y}, \tau\right) d \tau
$$

Talking Laplace Transform both sides of the Equation (4.10) and using the convolution property of the Laplace integral transform gives Equation (4.11)

$$
C(x, y, q)=\int_{0}^{\infty} e^{-q t}\left(\int_{0}^{T} w(\tau) G_{1}\left(x, y, t ; S_{x}, S_{y}, \tau\right) d \tau\right) \mathrm{dt}=w(q) G_{1}\left(x, y, t ; S_{x}, S_{y}, q\right)
$$

Making $w(q)$ subject of the formulae and talking inverse Laplace transform in the Equation (4.11) gives the result.

\subsubsection{Calculation of the Inverse Laplace Transform}

In this section, we apply the SLPs method to approximate Equation (4.6). The result of this section is summarized in Theorem 4.4

Theorem 4.4. Let $Q(r, p, q)$ be a continuous function composition of the three-dimension Laplace transform given by the Equation (4.12)

$$
Q(r, p, q)=\left(\frac{\zeta(r, p, q) \cdot p \cdot q \cdot r}{R_{1} G_{2}(x, y, t ; r, p, q)}\right)
$$

Let $Q(x, y, t)$ be its inverse transform. The inverse Laplace transform of Equation (4.6) 
is approximated by the Equation (4.13)

$$
C(x, y, t)=\sum_{i=0}^{N_{1}-1} \sum_{i=0}^{N_{2}-1} \sum_{i=0}^{N_{3}-1} \alpha_{i, j, k} \exp \left(a_{1} x+a_{2} y+a_{3} t\right) Z_{i}(x) Z_{j}(y) Z_{k}(t)
$$

where $\exp \left(a_{1} x+a_{2} y+a_{3} t\right)$ is the weighting function and the SLPs expansion coefficient $\alpha_{i, j, k}$ is given by Equation (4.14)

$$
\begin{gathered}
\alpha_{i, j, k}=\frac{(2 i+1)(2 j+1)(2 k+1)}{T_{1} T_{2} T_{3}} \sum_{g_{1}=0}^{i} \sum_{g_{2}=0}^{j} \sum_{g_{3}=0}^{k} \sigma_{i, g_{1}} \sigma_{j, g_{2}} \sigma_{k, g_{3}} \\
Q\left(a_{1}+\frac{g_{1}+1}{T}, a_{2}+\frac{g_{1}+1}{T}, a_{3}+\frac{g_{1}+1}{T}\right)
\end{gathered}
$$

where $T_{1}, T_{2}$ and $T_{3}$ are the arbitrary constants, and the elements $\sigma_{i, g_{1}}, \sigma_{j, g_{2}}$, and $\sigma_{k, g_{3}}$ are computed based on Equation (2.36).

Proof. Consider the Laplace transformation in three variables given by

$$
Q(r, p, q)=\int_{0}^{\infty} \int_{0}^{\infty} \int_{0}^{\infty} e^{-r x-p y-q t} Q(x, y, t) \mathrm{dxdydt}
$$

Using the SLPs, the inverse Laplace transform of $Q(r, p, q)$ is approximated by Equation (4.16):

$$
Q(x, y, t)=\sum_{i=0}^{N_{1}-1} \sum_{i=0}^{N_{2}-1} \sum_{i=0}^{N_{3}-1} \alpha_{i, j, k} \exp \left(a_{1} x+a_{2} y+a_{3} t\right) Z_{i}(x) Z_{j}(y) Z_{k}(t)
$$

where $\exp \left(a_{1} x+a_{2} y+a_{3} t\right)$ is the weighting function and the SLPs expansion coefficient $\alpha_{i, j, k}$ is obtained by the minimization of the integrable mean square error as in Equation (2.33). Thus the minimum is given by Equation (4.17)

$$
\begin{aligned}
\alpha_{i, j, k}= & \frac{(2 i+1)(2 j+1)(2 k+1)}{T_{1} T_{2} T_{3}} \int_{0}^{\infty} \int_{0}^{\infty} \int_{0}^{\infty} \exp \left(-\left(a_{1}+1 / T_{1}\right) x-\left(a_{2}+1 / T_{2}\right) y\right. \\
& \left.-\left(a_{3}+1 / T_{3}\right) t\right) Q(x, y, t) Z_{i}(x) Z_{j}(y) Z_{k}(t) \mathrm{dxdy} \mathrm{dt}
\end{aligned}
$$

Using Equations (2.29), (2.30), (2.36) and (4.16), Equation (4.17) is approximated by Equation (4.18)

$$
\begin{gathered}
\alpha_{i, j, k}=\frac{(2 i+1)(2 j+1)(2 k+1)}{T_{1} T_{2} T_{3}} \sum_{g_{1}=0}^{i} \sum_{g_{2}=0}^{j} \sum_{g_{3}=0}^{k} \sigma_{i, g_{1}} \sigma_{j, g_{2}} \sigma_{k, g_{3}} \\
Q\left(a_{1}+\frac{g_{1}+1}{T}, a_{2}+\frac{g_{1}+1}{T}, a_{3}+\frac{g_{1}+1}{T}\right)
\end{gathered}
$$

where the terms $\sigma_{i, g_{1}} \sigma_{j, g_{2}} \sigma_{k, g_{3}}$ are computed based on Equation (2.36). Comparison of 
the function formulation of $Q(r, p, q)$ and the function $C(x, y, t)$ confirms the proof.

\subsection{Localization of the source position}

In this section we use an iterative method to determine the source position $\left(S_{x}, S_{y}\right)$. For that we have the Theorem 4.5.

Theorem 4.5. Let $\mathbf{V}^{\perp}=\left(-v_{2}, v_{1}\right)^{T}$ be the perpendicular vector to $\mathbf{V}$ and let the functions, $\psi(x, y), \psi(x, y)^{\perp} \in L^{2}(\Omega)$, be two dispersion current functions defined in $\Omega$. Provided that $\psi(x, y)$, and $\psi(x, y)^{\perp}$ satisfy the Equations (4.19) and (4.20);

$$
\begin{aligned}
D \nabla \psi+\mathbf{V} & =0 \\
D \psi^{\perp}+\mathbf{V}^{\perp} & =0
\end{aligned}
$$

then the source position in the Equation (2.16) is given by the Equation (4.21);

$$
\mathbf{S}^{k+1}=\mathbf{S}^{k}+\frac{a_{L}^{2}}{\|\mathbf{V}\|^{2}}\left(\begin{array}{c}
v_{1}\left(\mathbf{S}^{k}\right)\left(\psi\left(\mathbf{S}^{k}\right)-\ln \left(\frac{P_{e}}{P_{0}}\right)\right)-v_{2}\left(\mathbf{S}^{k}\right)\left(\psi^{\perp}\left(\mathbf{S}^{k}\right)-\frac{P_{\psi^{\perp}}}{P_{0}}\right) \\
v_{2}\left(\mathbf{S}^{k}\right)\left(\psi\left(\mathbf{S}^{k}\right)-\ln \left(\frac{P_{e} \psi}{P_{0}}\right)\right)+v_{1}\left(\mathbf{S}^{k}\right)\left(\psi^{\perp}\left(\mathbf{S}^{k}\right)-\frac{P_{\psi^{\perp}}}{P_{0}}\right)
\end{array}\right)
$$

where the coefficients $P_{e}, P_{\psi^{\perp}}$, and $P_{0}$ are given by the Equations (4.22) to (4.24);

$$
\begin{aligned}
P_{0} & =\int_{0}^{T} w(t) e^{R_{1} t} \mathrm{dt} \\
P_{e^{\psi}} & =e^{R_{1} T} \int_{\Omega} e^{\psi(x, y)} c(x, y, T) \mathrm{dxdy}-\int_{0}^{T} \int_{\Gamma_{1}} e^{R_{1} t+\psi} D \nabla c \cdot \mathrm{d} \Gamma \mathrm{dt} \\
P_{\psi^{\perp}} & =e^{R_{1} T} \int_{\Omega} \psi^{\perp}(x, y) c(x, y, T) \mathrm{dx} \mathrm{dy}+\int_{0}^{T} \int_{\Gamma_{4}} c e^{R_{1} t}\left[\psi^{\perp} \mathbf{V}-D \mathbf{V}^{\perp}\right] \cdot \mathrm{d} \Gamma \mathrm{dt} \\
& -\int_{0}^{T} \int_{\Gamma_{L}} c e^{R t} D \mathbf{V}^{\perp} \cdot \mathrm{d} \Gamma \mathrm{dt}-\int_{0}^{T} \int_{\Gamma_{1}} e^{R t} \psi^{\perp} D \nabla c \cdot \mathrm{d} \Gamma \mathrm{dt}
\end{aligned}
$$

Proof. Using the Equations (2.11) to (2.13), the determinant of the matrix $D$ is given by $\operatorname{det}(D)=a_{L} a_{T} \neq 0$. Since the determinant of $D$ is nonzero, then $D$ is invertible. Therefore, there exists a unique vector field, $A$, which is a solution to the linear system 
$D A+\mathbf{V}=0$. Moreover, we have Equation (4.25):

$$
D^{-1}=\frac{1}{\operatorname{det}(D)}\left(\begin{array}{cc}
D_{y y} & -D_{x y} \\
-D_{y x} & D_{x x}
\end{array}\right) \Rightarrow A=\frac{-1}{a_{L} a_{T}}\left(\begin{array}{c}
D_{y y} v_{1}-D_{x y} v_{2} \\
D_{x x} v_{2}-D_{y x} v_{1}
\end{array}\right)=-\frac{\mathbf{V}}{a_{L}}
$$

Using Equation (2.5), it implies that $A$ is a gradient field derived from the scalar potential $\psi(x, y)$ that solves Equation (4.26);

$$
D \nabla \psi+\mathbf{V}=0
$$

Similarly, using the vector $\mathbf{V}^{\perp}=\left(-v_{2}, v_{1}\right)^{T}$, we have the function, $\psi^{\perp}(x, y)$ that solves Equation (4.27);

$$
D \nabla \psi^{\perp}+\mathbf{V}^{\perp}=0
$$

For $(a, b) \in \Omega$, the condition $\psi(a, b)=\psi^{\perp}(a, b)=0$ gives the solutions of the Equations (4.26) and (4.27) by the Equations (4.28) and (4.29) respectively.

$$
\begin{aligned}
\psi(x, y) & =-\int_{a}^{x} \frac{1}{a_{L}} v_{1}(\eta, y) \mathrm{d} \eta-\int_{b}^{y} \frac{1}{a_{L}} v_{2}(x, \xi) \mathrm{d} \xi \\
\psi(x, y)^{\perp} & =\int_{a}^{x} \frac{1}{a_{T}} v_{2}(\eta, y) \mathrm{d} \eta-\int_{b}^{y} \frac{1}{a_{T}} v_{1}(x, \xi) \mathrm{d} \xi
\end{aligned}
$$

Using the Equations (4.28) and (4.29), define the functions in the Equation (4.30);

$$
z(x, y, t)=e^{R t} u(x, y) \text { for } u(x, y)=\left\{e^{\psi}, \psi^{\perp}\right\}
$$

which are solutions to the homogeneous adjoint Equation (4.31);

$$
-\frac{\partial z}{\partial t}-\mathbf{V} \cdot \nabla z-\nabla \cdot D \nabla z+R_{2} z=0
$$

Multiply Equation (2.16) by $z(x, y, t)$ and integrate by parts over $\Omega \times(0, T)$ with the 
boundary conditions Equations (2.17) to (2.19) gives the Equation (4.32);

$$
\begin{aligned}
\int_{0}^{T} w(t) z\left(S_{x}, S_{y}, t\right) \mathrm{dt} & =\int_{\Omega} c(x, y, T) z(x, y, T) \mathrm{dx} \mathrm{dy} \\
& +\int_{0}^{T} \int_{\Gamma_{4}} c[z \mathbf{V}+D \nabla z] \cdot \mathrm{d} \Gamma \mathrm{dt}+\int_{0}^{T} \int_{\Gamma_{L}} c D \nabla z \cdot \mathrm{d} \Gamma \mathrm{dt} \\
& -\int_{0}^{T} \int_{\Gamma_{1}} z D \nabla c \cdot \mathrm{d} \Gamma \mathrm{dt}
\end{aligned}
$$

where on the lateral boundary, Equation (2.4), has been used. From substituting Equation (4.30) into the Equation (4.32), it follows that the unknown element $\left(S_{x}, S_{y}\right)$ defining the position of the source is given by Equation (4.33);

$$
\begin{aligned}
u\left(S_{x}, S_{y}\right) \int_{0}^{T} w(t) e^{R t} \mathrm{dt} & =e^{R T} \int_{\Omega} c(x, y, T) u(x, y) \mathrm{dx} \mathrm{dy} \\
& +\int_{0}^{T} \int_{\Gamma_{4}} c e^{R t}[u \mathbf{V}+D \nabla u] \cdot \mathrm{d} \Gamma \mathrm{dt}+\int_{0}^{T} \int_{\Gamma_{L}} c e^{R t} D \nabla u \cdot \mathrm{d} \Gamma \mathrm{dt} \\
& -\int_{0}^{T} \int_{\Gamma_{1}} e^{R t} u D \nabla c \cdot \mathrm{d} \Gamma \mathrm{dt}
\end{aligned}
$$

Using Theorem 4.3, the intensity function on the left-hand side of the Equation (4.33) is known. By substituting $u(x, y)=\left\{e^{\psi}, \psi^{\perp}\right\}$ in the Equation (4.33), a non-linear system of the Equation (4.34) results.

$$
\left\{\begin{array}{l}
\psi\left(S_{x}, S_{y}\right)=\ln \left(\frac{P_{e} \psi}{P_{0}}\right) \\
\psi^{\perp}\left(S_{x}, S_{y}\right)=\frac{P_{\psi^{\perp}}}{P_{0}}
\end{array}\right.
$$

where the coefficients, $P_{e} \psi, P_{0}$ and $P_{\psi^{\perp}}$ in the Equation (4.34) are given by the Equations (4.22) to (4.24). Newton's method is used on the Equation (4.34) to determine the zero of the associated vector function. Using Equations (4.26) and (4.27) for the expressions $\nabla \psi$ and $\nabla \psi^{\perp}$ respectively, the Jacobian matrix $J_{\Lambda}$ associated to the vector 
function $\Lambda=\left(\psi, \psi^{\perp}\right)^{T}$ is given by the Equation (4.35);

$$
J_{\Lambda}=\left(\begin{array}{cc}
\frac{\partial \psi}{\partial x} & \frac{\partial \psi}{\partial y} \\
\frac{\partial \psi^{\perp}}{\partial x} & \frac{\partial \psi^{\perp}}{\partial y}
\end{array}\right)=\frac{1}{a_{L}}\left(\begin{array}{cc}
-v_{1} & -v_{2} \\
v_{2} & -v_{1}
\end{array}\right)
$$

The determinant, $\operatorname{det}\left(J_{\Lambda}\right)$ of the $2 \times 2$ Jacobian matrix is given by the Equation (4.36);

$$
\operatorname{det}\left(J_{\Lambda}\right)=\frac{\partial \psi}{\partial x} \frac{\partial \psi^{\perp}}{\partial y}-\frac{\partial \psi}{\partial y} \frac{\partial \psi^{\perp}}{\partial x}=\frac{\|\mathbf{V}\|^{2}}{a_{L}^{2}}>0
$$

Thus the $2 \times 2$ Jacobian matrix is invertible in $\Omega$ and the inverse of the Jacobian matrix is given by Equation (4.37);

$$
J_{\Lambda}^{-1}=\frac{a_{L}^{2}}{\|\mathbf{V}\|^{2}}\left(\begin{array}{cc}
-v_{1} & v_{2} \\
-v_{2} & -v_{1}
\end{array}\right)
$$

Given an initial guess, $\left(S_{x}, S_{y}\right)$, for $k>0$, Equation (4.37) leads to perform the Newton's iterations to determine the unique solution of the Equation (4.34). We iterate Equation (4.38)

$$
\left(S_{x}^{k+1}, S_{y}^{k+1}\right)=\left(S_{x}^{k}, S_{y}^{k}\right)-J_{\Lambda}^{-1}\left(S_{x}^{k}, S_{y}^{k}\right)\left(\begin{array}{c}
\psi\left(S_{x}^{k}, S_{y}^{k}\right)-\ln \left(\frac{P_{e} \psi}{P_{0}}\right) \\
\psi^{\perp}\left(S_{x}^{k}, S_{y}^{k}\right)-\frac{P_{\psi^{\perp}}}{P_{0}}
\end{array}\right)
$$

Substitution of Equation (4.37) into Equation (4.38) with $\mathbf{S}^{k}=\left(S_{x}^{k}, S_{y}^{k}\right)$ gives Equation (4.21).

Then, for the clarity of our presentation we summarize the different steps of the established identification method in the Algorithm 1 


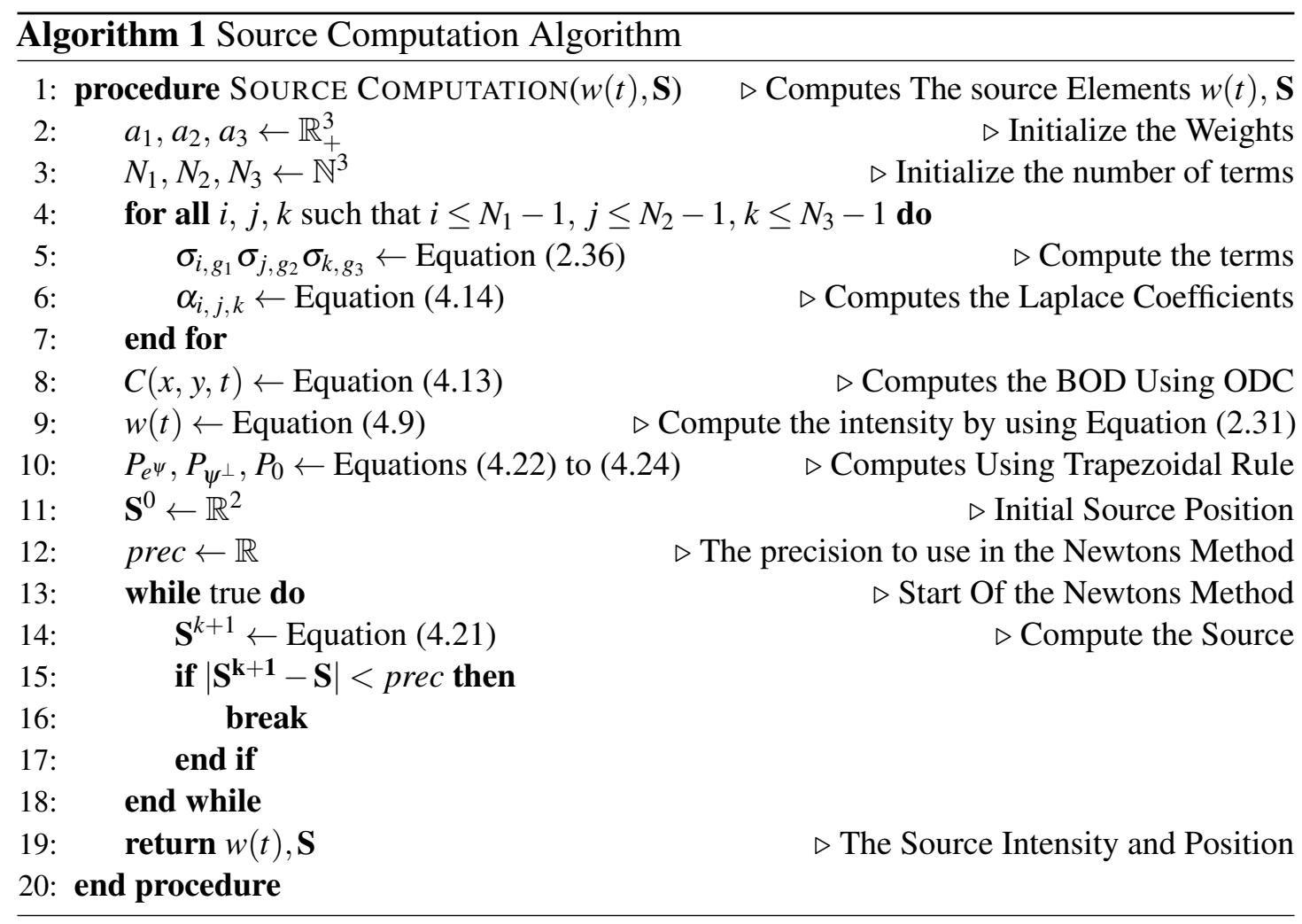

\section{NUMERICAL RESULTS AND DISCUSSION}

In this section, we carry out numerical experiments in the case of a square domain defined by the Equation (5.1):

$$
\Omega=\{(x, y) \text { such that } \quad 0<x<L \quad \text { and } \quad 0<y<L\}
$$

For the numerical computation, the domain $\Omega$ is scaled up according to the non-dimensional variables in the Equation (5.2):

$$
\tilde{x}=\frac{x}{L}, \tilde{y}=\frac{y}{L}, \tilde{S}=\frac{S}{L}, \tilde{\mathbf{V}}=\frac{\mathbf{V}}{L}, \tilde{D}=\frac{D}{L^{2}}, \tilde{C}=C, \tilde{w}=\frac{w}{L^{2}}, \tilde{R}=R, \tilde{t}=t
$$

Substituting the variables Equation (5.2) in Equations (2.15) to (2.19) gives Equations (5.3) to (5.7);

$$
\begin{aligned}
\frac{\partial \tilde{\zeta}}{\partial \tilde{t}}+\tilde{\mathbf{V}} \cdot \tilde{\nabla} \tilde{\zeta}-\tilde{\nabla} \cdot(\tilde{D} \tilde{\nabla} \tilde{\zeta})+\tilde{R}_{1} \tilde{\zeta} & =\tilde{R}_{1} \tilde{C} \\
\frac{\partial \tilde{C}}{\partial \tilde{t}}+\tilde{\mathbf{V}} \cdot \tilde{\nabla} \tilde{C}-\tilde{\nabla} \cdot(\tilde{D} \tilde{\nabla} \tilde{C})+\tilde{R}_{2} \tilde{C} & =\tilde{w}(\tilde{t}) \delta\left(\tilde{x}-\tilde{S}_{x}, \tilde{y}-\tilde{S}_{y}\right) \\
\tilde{C}(\tilde{x}, \tilde{y}, 0)=\tilde{\zeta}(\tilde{x}, \tilde{y}, 0) & =0 \quad \text { in } \quad \Omega \\
\tilde{C}(\tilde{x}, \tilde{y}, \tilde{t})=\tilde{\zeta}(\tilde{x}, \tilde{y}, \tilde{t}) & =0 \quad \text { on } \quad \Gamma_{1} \times(0, T) \\
\tilde{\nabla} C(\tilde{x}, \tilde{y}, \tilde{t})=\tilde{\nabla} \zeta(\tilde{x}, \tilde{y}, \tilde{t}) & =0 \quad \text { on } \quad\left(\Gamma_{L} \cup \Gamma_{4}\right) \times(0, T)
\end{aligned}
$$


We employ in Equations (5.3) to (5.7) the coefficients in Equation (5.8)

$$
L=1000 m, a_{T}=10 m, a_{L}=0.2 m, \tilde{R}=2.2 \times 10^{-6} s^{-1}
$$

For the velocity field, we employ the field defined in $\Omega$ by Equation (5.9)

$$
\mathbf{V}=\left\{\begin{array}{l}
-\left(\beta e^{\left(\frac{\pi x}{L}\right)}-\alpha e^{\left(-\frac{\pi x}{L}\right)}\right) \bar{V} \cos \left(\frac{\pi y}{L}\right)+V_{0} \\
\left(\beta e^{\left(\frac{\pi x}{L}\right)}+\alpha e^{\left(-\frac{\pi x}{L}\right)}\right) \bar{V} \sin \left(\frac{\pi y}{L}\right)
\end{array}\right.
$$

where $\beta, \alpha, V_{0}$, and $\bar{V}$ are real numbers. The velocity in the Equation (5.9) satisfies the required conditions introduced in the Equations (2.3) and (2.5). Using $a=b=0$ and making substitution of Equation (5.9) into the Equations (4.28) and (4.29), the dispersion current functions are given by the Equations (5.10) and (5.11)

$$
\begin{aligned}
\psi & =\frac{L}{\pi a_{L}}\left[-V_{0} \pi x-\left(\left(\alpha+\beta-2(\alpha+\beta) e^{\left(\frac{\pi x}{L}\right)}\right) \cos \left(\frac{\pi y}{L}\right)+(\alpha+\beta) e^{\left(\frac{\pi x}{L}\right)}\right) \bar{V}\right] \\
\psi^{\perp} & =\frac{L}{\pi a_{T}}\left[-V_{0} \pi y+\left(\alpha-\beta-2(\alpha-\beta) e^{\left(\frac{\pi x}{L}\right)}\right) \bar{V} \sin \left(\frac{\pi y}{L}\right)\right]
\end{aligned}
$$

As for the non-dimensional variables in the Equations (5.3) to (5.7), we employ uniform mesh sizes $\Delta x=N_{x}$ and $\Delta y=1 / N_{y}$ with a constant time-step $\Delta t=1 / N_{t}$ and use Finite Volume Method (FVM). To carry out numerical experiments, in the Equation (5.9), we employ the coefficients $V_{0}=0.70, \bar{V}=10^{-2}, \alpha=1.0$ and $\beta=\exp (100 \pi)$. We use a mesh with $N_{x}=100$ and $N_{y}=10$ whereas $N_{t}=180$. To generate the boundary records $d_{a}(t), d_{b}(t)$ introduced in Equation (2.22), we solve Equations (5.3) to (5.7) using FVM, where the time-dependent intensity function is defined by Equation (5.12);

$$
w(t)=\sum_{i=1}^{3} b_{i} e^{-u_{i}\left(t-q_{i}\right)^{2}}
$$

where $b_{1}=1.2, b_{2}=0.4, b_{3}=0.6, u_{1}=10^{-6}, u_{2}=5 \times 10^{-5}, u_{3}=10^{-6}, q_{1}=$ $4.5 \times 10^{3}, q_{2}=6.5 \times 10^{3}, q_{3}=9 \times 10^{3}$. The intensity function is monitored for a period of $T=14400$ s (4hours). For the simulation we use the mean value of the intensity function defined by Equation (5.13);

$$
\frac{1}{T} \int_{0}^{T} w(t) \mathrm{dt}=\frac{\sqrt{\pi}}{2 T} \sum_{k=1}^{3} \frac{b_{k}}{\sqrt{u_{k}}}\left[\operatorname{erf}\left(\sqrt{u_{k}}\left(t-q_{k}\right)\right)-\operatorname{erf}\left(-\sqrt{u_{k}} q_{k}\right)\right]
$$

where

$$
\operatorname{erf}(\theta)=\int_{0}^{\theta} e^{-s^{2}} \mathrm{ds}
$$


is the Gauss error function. As far as the source position $S=(S x, S y)$ is concerned, we employ the approximation of the Dirac mass in Equation (5.14) [5];

$$
\delta(x-S x, y-S y) \approx\left(\frac{1+\cos (\pi(x-S x))}{2 \varepsilon}\right)\left(\frac{1+\cos (\pi(y-S y))}{2 \varepsilon}\right)
$$

We set the parameter, $\varepsilon=10^{-5}$ in Equation (5.14). Theorem 2.1 allows us to uniquely identify the unknown elements $S_{x}, S_{y}$ and $w(t)$ defining $F$ in the Equation (2.14) that generated the observations Equation (2.21). In the sequel, we present numerical solution of the dimensionless problem Equations (5.3) to (5.7) obtained using the FVM. The numerical results presented in Table 5.1 show the simulated BOD and ODC used for the source identification. In the last column of Table 5.1, we compute the absolute differences of the simulated BOD and ODC that is abs(BOD - ODC).

Table 5.1: Differences between simulated BOD and ODC

\begin{tabular}{|l|r|r|r|}
\hline & Simulated Oxygen Deficit & Simulated BOD Data & Absolute Differences \\
\hline 1 & 0.095527 & 0.087149 & 0.008377 \\
2 & 0.075835 & 0.068769 & 0.007066 \\
3 & 0.061037 & 0.055357 & 0.005680 \\
4 & 0.050232 & 0.045578 & 0.004654 \\
5 & 0.042355 & 0.038449 & 0.003906 \\
6 & 0.036614 & 0.033254 & 0.003360 \\
7 & 0.032431 & 0.029468 & 0.002963 \\
8 & 0.029382 & 0.026709 & 0.002673 \\
9 & 0.027162 & 0.024700 & 0.002462 \\
10 & 0.025544 & 0.023236 & 0.002309 \\
11 & 0.024366 & 0.022170 & 0.002197 \\
12 & 0.023509 & 0.021393 & 0.002115 \\
13 & 0.022884 & 0.020828 & 0.002056 \\
14 & 0.022429 & 0.020416 & 0.002013 \\
15 & 0.022098 & 0.020117 & 0.001981 \\
\hline
\end{tabular}


To identify the source, we apply the detection-identification method developed in the previous sections by following the steps described in Algorithm 1. To this end, we initialize the weights $a_{1}=0.05, a_{2}=0.4, a_{3}=4$ and the number of terms for the summation $N_{1}=100, N_{2}=10, N_{3}=180$. The weights and the number of terms are used in the Loop to calculate the coefficients in the lines 4 to 6 of the Algorithm 1.

To compute the BOD using ODC, we first compute the Green's Function and the $\zeta(x, y, t)$ for Equations (5.3) to (5.7) using FVM by solving Equations (3.1) to (3.5) and Equations (5.3) to (5.7) respectively. Then we use Equation (4.12) of which the BOD is completely determined by step 8 of the Algorithm 1. Figure 5.1 shows the numerical solution of the Green's function equation.

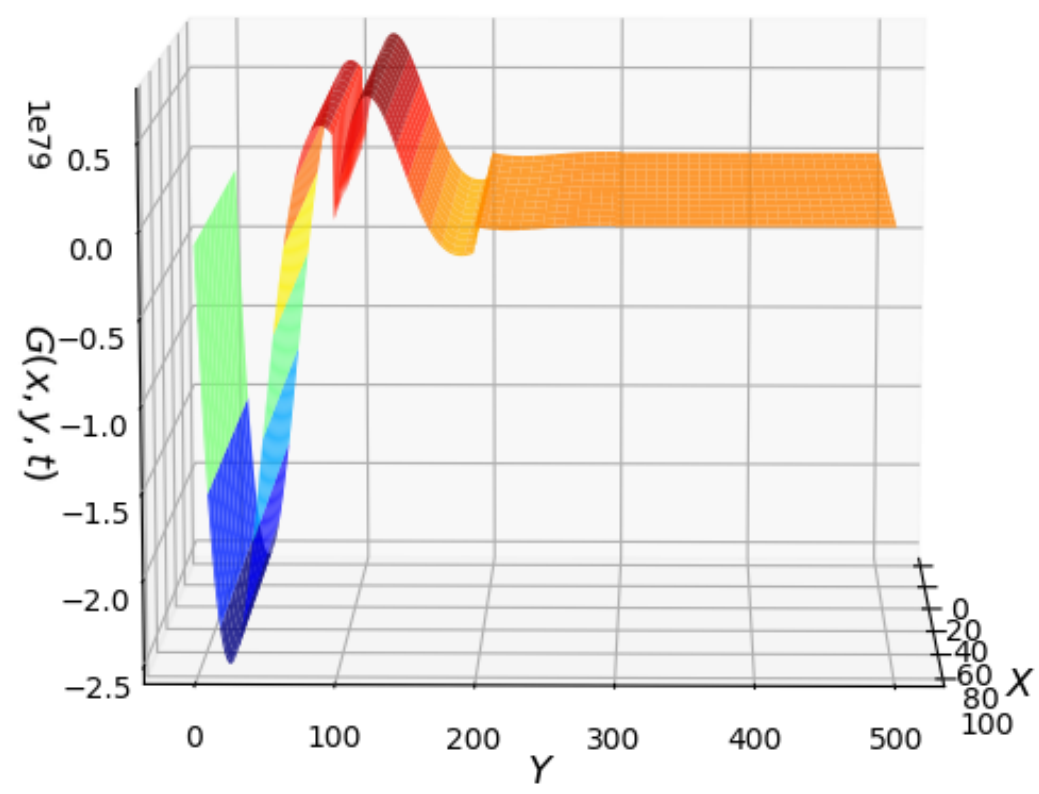

Figure 5.1: The Green's Function For the System

The intensity function $w(t)$ is computed from step 9 of the Algorithm 1. Finally, to compute the source position, the integrals $P_{e^{\psi}}, P_{\psi^{\perp}}, P_{0}$ are computed using trapezoidal rule in step 10 of the Algorithm 1. Using 10 initial source positions, $\mathbf{S}^{0}$, and a precision of 0.01 , we compute the source positions using the Newtons Method as outlined in lines 13 to 18 of the Algorithm 1. Figures 5.2 to 5.7 shows the computed intensity functions at various source positions. 


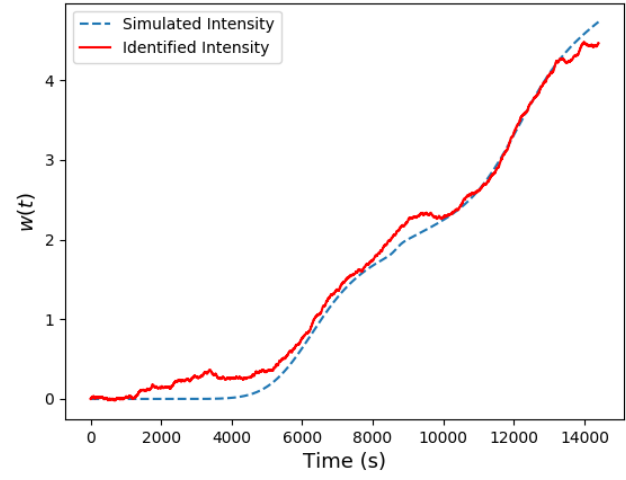

Figure 5.2: Intensity Source at $(100,100)$

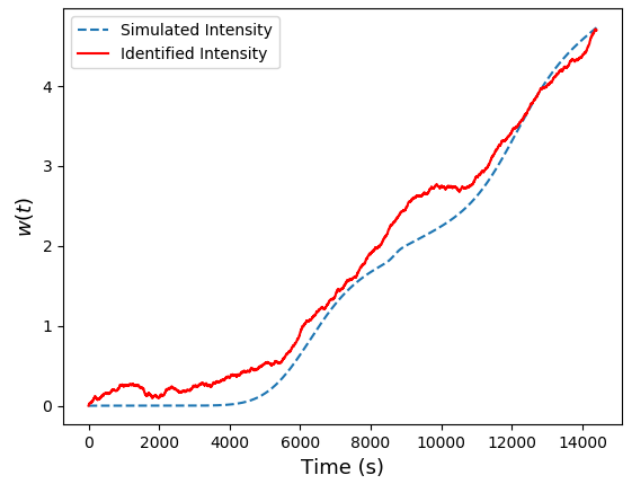

Figure 5.4: Intensity Source at $(600,450)$

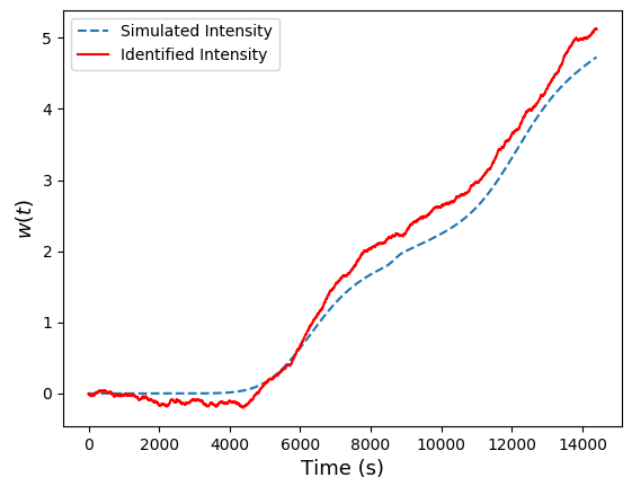

Figure 5.6: Intensity Source at $(1000,90)$

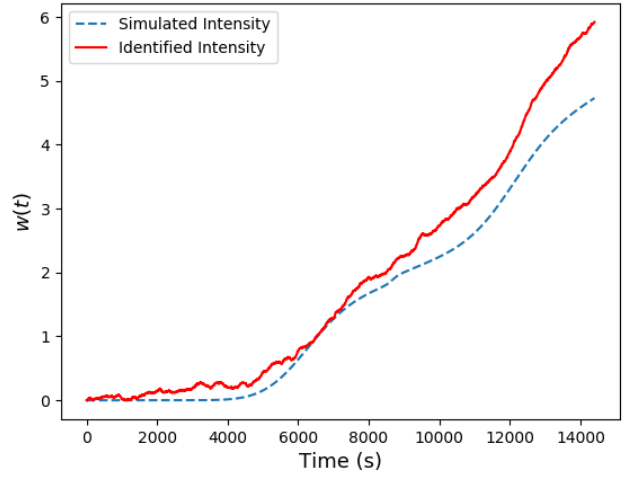

Figure 5.3: Intensity Source at $(200,80)$

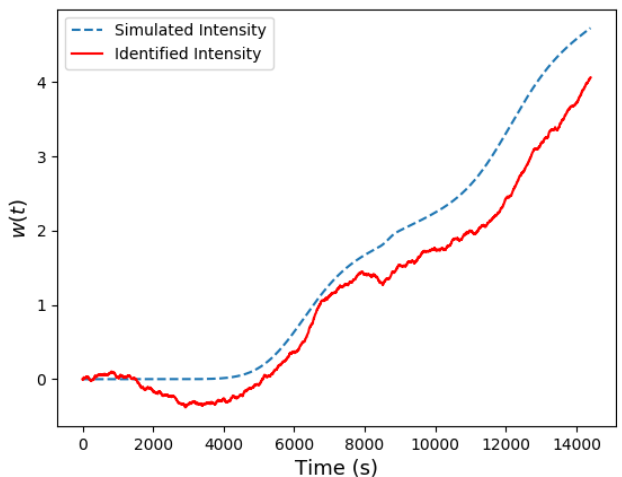

Figure 5.5: Intensity Source at $(80,800)$

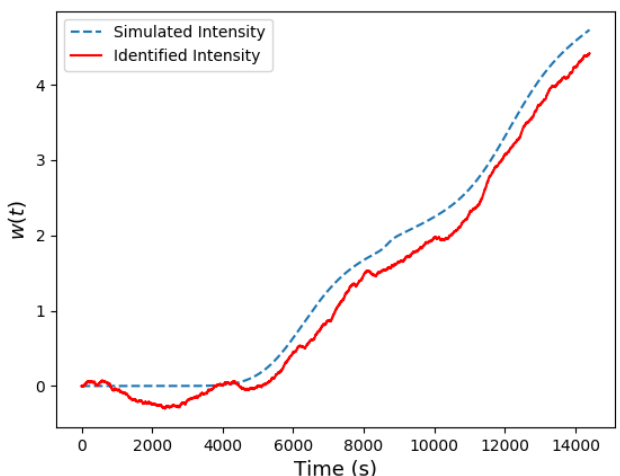

Figure 5.7: Intensity Source at $(80,1200)$ 
In Table 5.2 we give the recovered source positions corresponding to different initial source positions

Table 5.2: Locations of Simulated Source Positions and Identified Source Positions

\begin{tabular}{|l|l|l|}
\hline & Used source position & Identified source position \\
\hline 1 & $\mathbf{S}=(80,800)$ & $\mathbf{S}=(83.5171,791.6173)$ \\
2 & $\mathbf{S}=(90,1000)$ & $\mathbf{S}=(90.0677,1010.8674)$ \\
3 & $\mathbf{S}=(100,1200)$ & $\mathbf{S}=(80.3909,1539.9904)$ \\
4 & $\mathbf{S}=(200,450)$ & $\mathbf{S}=(197.7977,451.4966)$ \\
5 & $\mathbf{S}=(300,80)$ & $\mathbf{S}=(299.959,76.353)$ \\
6 & $\mathbf{S}=(600,100)$ & $\mathbf{S}=(499.517,119.116)$ \\
7 & $\mathbf{S}=(800,60)$ & $\mathbf{S}=(790.5319,59.6309)$ \\
8 & $\mathbf{S}=(900,300)$ & $\mathbf{S}=(864.7572,289.6473)$ \\
9 & $\mathbf{S}=(1000,700)$ & $\mathbf{S}=(889.4533,644.9977)$ \\
10 & $\mathbf{S}=(1200,900)$ & $\mathbf{S}=(1433.1591,833.3395)$ \\
\hline
\end{tabular}

The numerical results presented in Table 5.2 show that the inverse source identification method established enables to identify the source position with very good accuracy. The observed error on the identified source position are due to the fact that BOD records are not generated by a point source, that is, Dirac delta function but rather by its approximation given in Equation (5.14). The second error is due to the approximation of integrals involved in the coefficients $P_{0}, P_{e^{\psi}}$ and $P_{\psi^{\perp}}$ found in Equations (4.22) to (4.24) respectively. However, for the identified source intensity function $w(t)$, the analysis of the numerical results presented in Figures 5.2 to 5.7 indicates that the error becomes more significant with time. This could be explained by the fact that $w(t)$ is identified from solving the deconvolution problem introduced in Equation (4.9). Therefore, for each $t_{i} \in(0, T)$, the identification of $w\left(t_{i}\right)$ is affected by the errors introduced on the used BOD.

\section{CONCLUSION}

A solution of an inverse source problem for identification of the time-dependent point source has been obtained in this study. The solution involved application of a system of two coupled 2D ADRE to groundwater source identification. Validation of the model was demonstrated by using hypothetical examples as there was no oxygen deficit data in the literature. To generate the hypothetical data FVM was used to solve the forward problem 
and approximation made at discreet points. Numerical experiments on the simulated BOD data were carried out. The obtained numerical results shows that the developed identification method is accurate. In this paper, we only considered the transport of the pollutant in the groundwater without considering groundwater flow. An outlook for the results established in the present study is their extension towards at least the following directions: Firstly, incorporating groundwater flow equations for the transport of groundwater, ADRE for the transport of the pollutants and solitary vibrations available on the interaction between groundwater and surface water. Secondly, apply the developed identification method using other reference geometries and real-life measurements taken on a flow crossing a monitored domain of arbitrary geometric shape. Thirdly, treat the three-dimensional case for the ADRE. Finally, apply the developed methodology into other applications.

\section{ACKNOWLEDGMENT}

This work was supported by Pan African University Institute for Basic Sciences Technology and Innovation (PAUSTI) for which the authors express their sincere gratitude.

\section{BIBLIOGRAPHY}

[1] Omega Soko Alpha, Verdiana Grace Masanja, and Okelo Jeconiah Abonyo. Identification of multiple unknown point sources occurring in the $2 \mathrm{~d}$ transport equation: application to groundwater pollution source identification. J. Math. Comput. Sci., 10(4):833-862, 2020.

[2] Jacob Bear and Yehuda Bachmat. Introduction to modeling of transport phenomena in porous media, volume 4. Springer Science \& Business Media, 2012.

[3] Joyati Debnath and RS Dahiya. Theorems on multidimensional laplace transform for solution of boundary value problems. Computers \& Mathematics with Applications, 18(12):1033-1056, 1989.

[4] William E Dobbins. Bod and oxygen relationship in streams. Journal of the Sanitary Engineering Division, 90(3):53-78, 1964.

[5] Björn Engquist, Anna-Karin Tornberg, and Richard Tsai. Discretization of dirac delta functions in level set methods. Journal of Computational Physics, 207(1): 28-51, 2005.

[6] Adel Hamdi. Identification of a time-varying point source in a system of two coupled linear diffusion-advection-reaction equations: application to surface water pollution. Inverse Problems, 25(11):115009, 2009.

[7] S Jouanneau, Loic Recoules, MJ Durand, Ali Boukabache, Vincent Picot, Y Primault, A Lakel, M Sengelin, B Barillon, and G Thouand. Methods for assessing biochemical oxygen demand (bod): A review. Water research, 49:62-82, 2014. 
[8] Jacques-Louis Lions. Pointwise control for distributed systems. Control Systems, pages 1-39, 1992.

[9] Imed Mahfoudhi. Problèmes inverses de sources dans des équations de transport à coefficients variables. PhD thesis, Rouen, INSA, 2013.

[10] Akira Okubo. Diffusion and ecological problems:(mathematical models). Biomathematics, 1980.

[11] Gerald T Orlob. Mathematical modeling of water quality: Streams, lakes and reservoirs, volume 12. John Wiley \& Sons, 1983.

[12] JB Passioura. Hydrodynamic dispersion in aggregated media: 1. theory. Soil science, 111(6):339-344, 1971.

[13] Jeffrey Rauch. Hyperbolic partial differential equations and geometric optics, volume 133. American Mathematical Soc., 2012.

[14] Jean-Claude Saut and Bruno Scheurer. Unique continuation for some evolution equations. Journal of differential equations, 66(1):118-139, 1987.

[15] Dong-her Shih, Ruey-Chyi Shen, and Tow-chen Shiau. Numerical inversion of multidimensional laplace transforms. International journal of systems science, 18 (4):739-742, 1987. 